Article

\title{
Well-Defined Diimine Copper(I) Complexes as Catalysts in Click Azide-Alkyne Cycloaddition Reactions
}

\author{
Jordi Markalain Barta ${ }^{\dagger}$ and Silvia Díez-González * \\ Department of Chemistry, Imperial College London, Exhibition Road, South Kensington, \\ SW7 2AZ London, UK; E-Mail: j_markalain@hotmail.com \\ $\dagger$ Erasmus Student from the Universidad de Barcelona, Barcelona 08130, Spain. \\ * Author to whom correspondence should be addressed; E-Mail: s.diez-gonzalez@imperial.ac.uk; \\ Tel.: +44-207-549-699.
}

Received: 29 June 2013; in revised form: 21 July 2013 / Accepted: 23 July 2013 /

Published: 26 July 2013

\begin{abstract}
A series of 1,4-disubstituted 1,2,3-triazoles have been prepared in high yields while respecting the stringent Click criteria. In these reactions, highly stable pre-formed complexes bearing diimine ligands were used.
\end{abstract}

Keywords: ligand; diimine; copper(I); Click; dipolar cycloaddition; azide

\section{Introduction}

The introduction of copper(I) catalysts in the cycloaddition of azides and terminal alkynes represents one of the latest success stories of organometallic catalysis [1-4]. Not only is this transformation high yielding and completely regioselective, but also it exemplifies the utility and importance of Click chemistry [5]. Whereas this cycloaddition reaction has been applied in a plethora of fields, the efforts to develop efficient catalytic systems, respectful of the Click criteria, have been significantly fewer. Nevertheless, the use of ligands in the cycloaddition of alkynes and azides has been shown to stabilize the copper(I) centre, increase the catalytic activity, and even modulate it [6]. Among the different families of ligands applied in this reaction, nitrogen-based ones are arguably the most widely used. Whereas Meldal et al. used diisopropylethylamine in their groundbreaking report [1], $N, N^{\prime}, N^{\prime \prime}$-pentametyletylentriamine (PMDETA) and tris-triazoles are also very popular choices [6]. However, examples of well-defined catalysts containing nitrogen based remain scarce [7-11]. Most 
reported examples employ polydentate ligands such as polytriazoles or tren ligands (Figure 1). Particularly relevant to this work are complexes bearing bis(aryl)acenaphthenenquinonediimine (Ar-BIAN) ligands $\mathbf{C}$. These have been shown to lead to the formation of triazoles in conversion ranging from modest to good in $\mathrm{THF}$ at $50{ }^{\circ} \mathrm{C}[11]$.

Figure 1. Reported preformed copper(I) catalysts with $N$-ligands.<smiles>OC1(c2cn(Cc3ccccc3)nn2)Cn2nc(Cn3cccc3)cc2C1(Cl)c1cn(Cc2ccccc2)nn1</smiles>

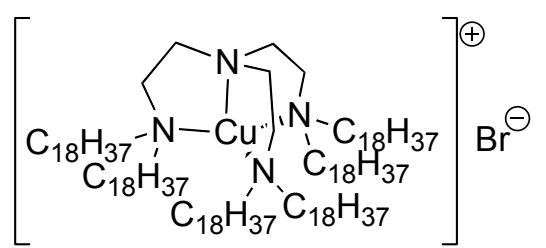

B

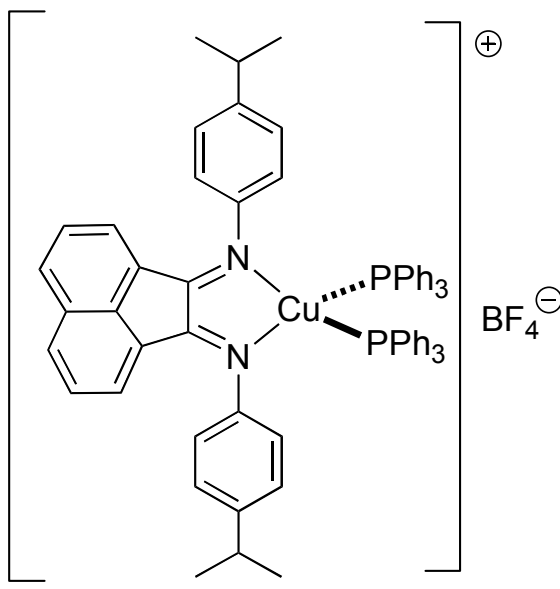

C

Herein, we report the catalytic activity of pre-formed copper(I) complexes bearing one or two $\alpha$-diimine ligands in the preparation of 1,2,3-triazoles from azides and terminal alkynes. The structures of the screened complexes are shown in Figure 2. These complexes are all highly stable and easy to prepare from simple diazabutadiene compounds [12].

Figure 2. Copper(I) catalysts used in this study.<smiles>CNC1(OC)N(c2c(C(C)C)cccc2C(C)C)C=CN1c1c(C(C)C)cccc1C(C)C</smiles>

1

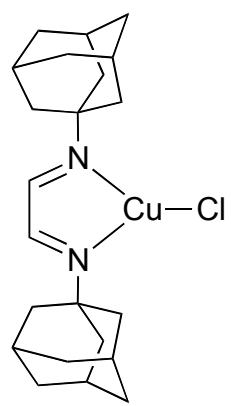

5<smiles>Cc1cc(C)c(N2C=CN(c3c(C)cc(C)cc3C)N([C](Cl)N(C=CN(c3c(C)cc(C)cc3C)c3c(C)cc(C)cc3C)c3c(C)cc(C)cc3C)C2)c(C)c1</smiles>

2

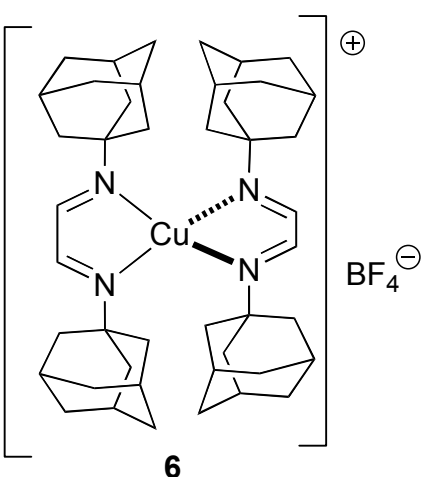

6<smiles>COc1ccc(N2C=CN(c3ccc(OC)cc3)[C](Cl)[C]2Cl)cc1</smiles>

3

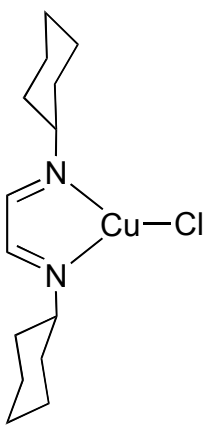

7<smiles></smiles><smiles></smiles> 


\section{Results and Discussion}

We started our optimization studies with the screening of different solvents. We chose cationic complex 1 and benzyl azide (9a) and phenylacetylene as the model reaction. Using $5 \mathrm{~mol} \%$ of the copper catalyst, virtually no conversion of the starting materials was observed with $\mathrm{MeCN}$, cyclohexane, or ethyl acetate, whereas poor results were obtained in $\mathrm{MeOH}$ or under neat conditions (Table 1). Satisfyingly, complete conversions into triazole 10a were obtained on water and in acetone (Table 1, entries 8 and 9). Similar results were observed in THF or in a mixture water $/ t-\mathrm{BuOH}$, however, reactions in these solvents were not always reproducible and they were not studied further (Table 1, entries 6 and 7).

In order to determine the best reaction medium for this reaction, the copper loading was next reduced to $2 \mathrm{~mol} \%$ with acetone and water (Table 1, entries 10 and 11). A higher conversion of $86 \%$ was observed in acetone and hence it was kept as solvent for the rest of the study. It is important to note that all tested solvents were technical grade, and in particular, the acetone employed here was the one normally used for cleaning the glassware in the laboratory.

Table 1. Solvent screening.

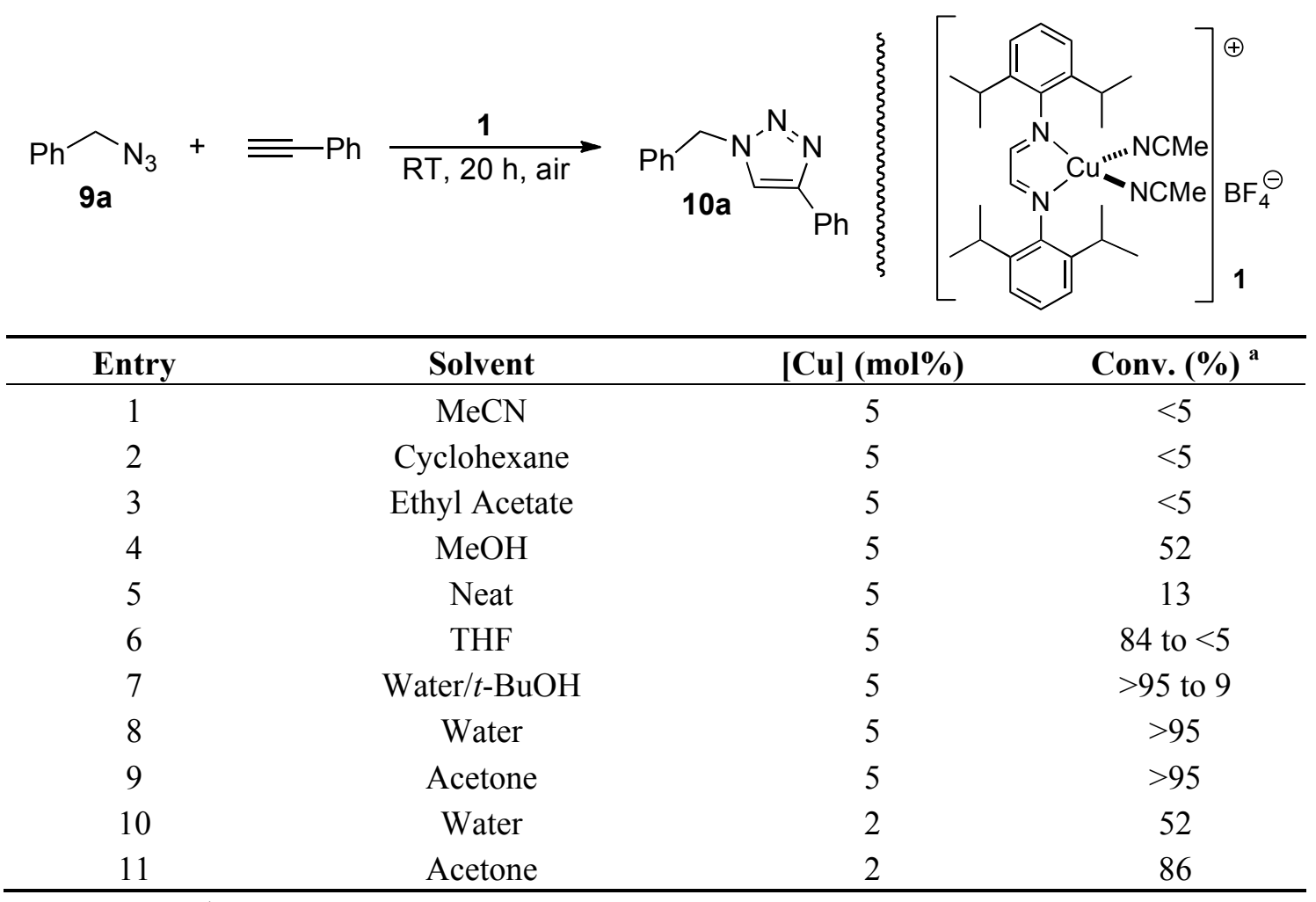

${ }^{\text {a } 1} \mathrm{H}-\mathrm{NMR}$ conversions are the average of at least two independent reactions.

We next performed a catalyst screening in acetone at room temperature using $2 \mathrm{~mol} \%$ of different diimine copper complexes (Scheme 1). All the tested complexes performed well in the model reactions with conversion ranging from $42 \%$ to complete. For complexes bearing aromatic groups on the ligands, steric hindrance proved to be more beneficial to the reaction outcome than the presence of electron donating groups. In general, cationic complexes performed better than their neutral analogues, except for complexes bearing adamantyl imine ligands 5 and 6. Additionally, the best performing 
complexes had aliphatic substituents on the diimine ligand(s). Hence, complexes 5, 7 and 8 led to total conversions and further experiments were run with these in order to determine the best performing one.

Scheme 1. Catalyst screening ${ }^{\text {a }}$.

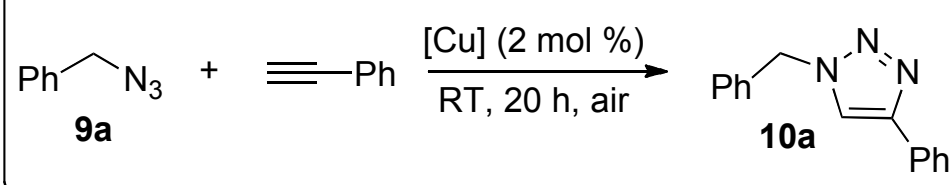

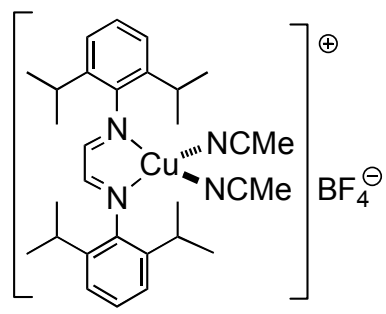

$1,86 \%$

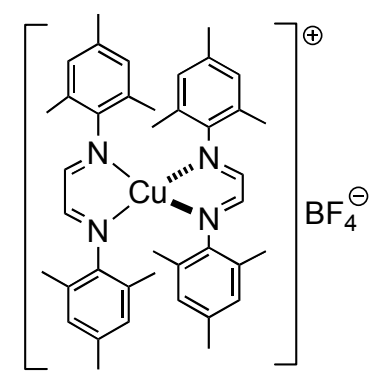

$2,82 \%$

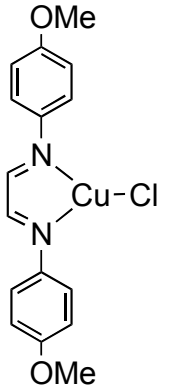

$3,42 \%$

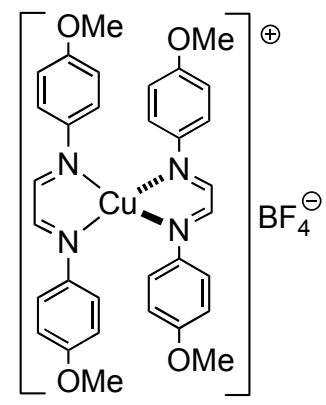

$4,68 \%$

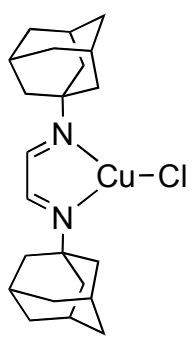

5, $<95 \%$

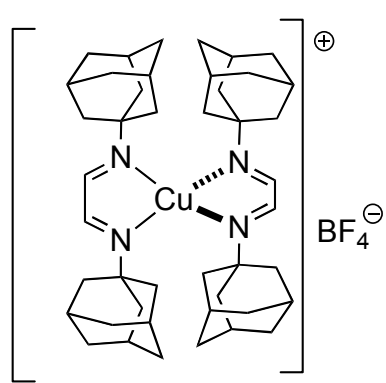

$6,57 \%$

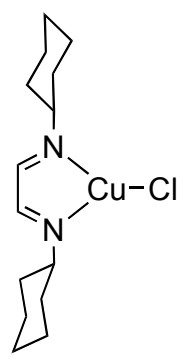

$7,<95 \%$

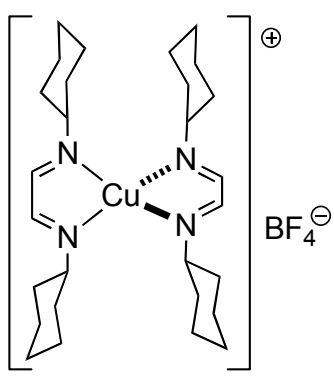

8, $<95 \%$

${ }^{\text {a } 1} \mathrm{H}-\mathrm{NMR}$ conversions are the average of at least two independent reactions.

First, the metal loading was further reduced (Table 2). Similar results were obtained with $1 \mathrm{~mol} \%$ $[\mathrm{Cu}]$, but at $0.5 \mathrm{~mol} \%[\mathrm{Cu}]$ it became apparent that complexes bearing either one or two cyclohexyl diimine ligands $\mathbf{7}$ and $\mathbf{8}$ displayed a better activity than complex $\mathbf{5}$, with adamantyl substituents. Gratifyingly, complex 8 could still achieve total conversion in a more concentrated reaction (1 M rather than $0.5 \mathrm{M}$ ), and it was chosen as the optimal catalyst within the tested series.

With an optimized catalytic system in hand, the scope of the reaction was then explored. All reactions were carried out in technical grade acetone, in air and in no cases oxidation to copper(II) or disproportionation to copper(0) and copper(II) were observed. Also, no other by-products were formed and the prepared triazoles could be easily isolated as pure products in high yields after a simple extraction (Scheme 2).

A number of functional groups were well tolerated, such as alcohols, amines, alkenes and nitriles. Benzyl, alkyl and aryl azides well suitable cycloaddition partners, as well as electron rich or electron poor alkynes. In some cases, total conversions were not reached under the optimized conditions. However, a slight increase in the reaction temperature (from RT to $40{ }^{\circ} \mathrm{C}$ ) or the metal loading was enough to ensure a high isolated yield. 
Table 2. Final optimization reactions.

\begin{tabular}{|c|c|c|c|}
\hline Entry & {$[\mathbf{C u}]$} & Concentration (M) & Conv. $(\%)^{\mathrm{a}}$ \\
\hline 1 & $5,1 \mathrm{~mol} \%$ & 0.5 & $<95$ \\
\hline 2 & 7, $1 \mathrm{~mol} \%$ & 0.5 & $<95$ \\
\hline 3 & $8,1 \mathrm{~mol} \%$ & 0.5 & $<95$ \\
\hline 4 & $5,0.5 \mathrm{~mol} \%$ & 0.5 & 63 \\
\hline 5 & $7,0.5 \mathrm{~mol} \%$ & 0.5 & 92 \\
\hline 6 & $8,0.5 \mathrm{~mol} \%$ & 0.5 & $<95$ \\
\hline 7 & $7,0.5 \mathrm{~mol} \%$ & 1.0 & 93 \\
\hline 8 & $8,0.5 \mathrm{~mol} \%$ & 1.0 & $<95$ \\
\hline
\end{tabular}

${ }^{\mathrm{a}}{ }^{1} \mathrm{H}-\mathrm{NMR}$ conversions are the average of at least two independent reactions.

Scheme 2. Preparation of 1,2,3-triazoles catalyzed by complex $\mathbf{8}^{\text {a }}$.

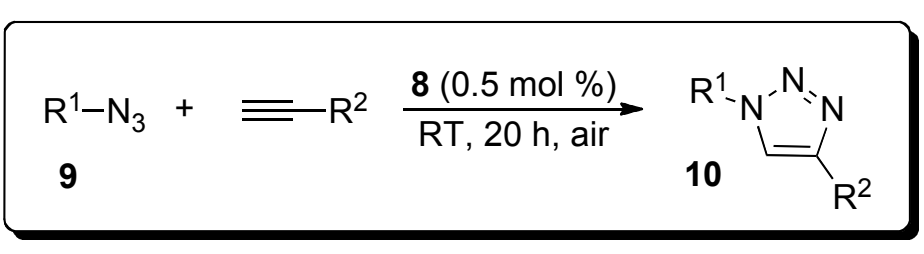<smiles>c1ccc(Cn2cc(-c3ccccc3)nn2)cc1</smiles>

10a, $95 \%$<smiles>Brc1cn(Cc2ccccc2)nn1</smiles>

$10 \mathrm{~b}, 92 \%$ b,c

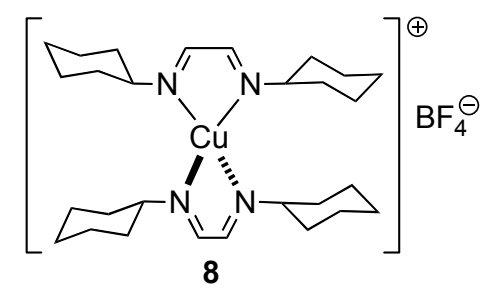<smiles>CC(C)N(CCn1cc(-c2ccccc2)nn1)C(C)C</smiles>

$10 d, 85 \%$<smiles>CCOC(=O)c1cn(CCC2OCCO2)nn1</smiles><smiles>CN(C)c1cn(CCc2ccccc2)nn1</smiles>

$10 f, 88 \%^{\mathrm{d}}$<smiles>N#CC1CC1n1cc(P)nn1</smiles>

$10 \mathrm{i}, 94 \%$<smiles>C(=C/c1ccccc1)\Cn1cc(-c2ccccc2)nn1</smiles>

$\operatorname{10g}, 96 \%$<smiles>CC(c1ccccc1)n1cc(C2CC2)nn1</smiles>

${ }^{\mathrm{a}}$ Isolated yields are the average of at least two independent reactions; ${ }^{\mathrm{b}} 2 \mathrm{~mol} \% \mathbf{8} ;{ }^{\mathrm{c}} 40{ }^{\circ} \mathrm{C} ;{ }^{\mathrm{d}} 1 \mathrm{~mol} \% \mathbf{8}$.

\section{Experimental Section}

\subsection{General}

All reagents were commercially available and used as received. ${ }^{1} \mathrm{H}-\mathrm{NMR}(400 \mathrm{MHz})$ and ${ }^{13} \mathrm{C}-\mathrm{NMR}$ $(100 \mathrm{MHz})$ spectra were recorded in $\mathrm{CDCl}_{3}$ on a Bruker AVANCE400 spectrometer at room 
temperature. Chemical shifts $(\delta)$ are reported in $\mathrm{ppm}$ and referenced to tetramethylsilane $\left({ }^{1} \mathrm{H}\right)$ and deuterated chloroform $\left({ }^{13} \mathrm{C}\right)$, respectively. All reactions were carried out in air and using technical solvents with no particular precautions to exclude oxygen or moisture. All reported yields are isolated yields and are the average of at least two independent reactions. Benzyl azide (9a) [13] 2-azidoethyldiisopropylamine (9b) [14], 2-(2-azidoethyl)-1,3-dioxolane (9c) [15], (2-azidoethyl)benzene (9d) [13], 3-(azidoprop-1-en-yl)benzene (9e) [16], phenyl azide (9f) [17], 6-azidohexanenitrile (9g) [18] and (1-azidoethy)benzene (9h) [13] are known in the literature and the corresponding spectroscopic data for all these compounds were in good agreement with the reported data.

\subsection{Catalytic Results}

General Procedure for the [3+2] Cycloaddition of Azides and Terminal Alkynes

In a vial fitted with a screw cap, azide $9(1 \mathrm{mmol})$, alkyne $(1 \mathrm{mmol})$, acetone $(1 \mathrm{~mL})$ and 8 ( $3 \mathrm{mg}$, $0.5 \mathrm{~mol} \%)$ were loaded. The reaction was allowed to proceed at room temperature overnight $(20 \mathrm{~h})$. The reaction mixture was then hydrolyzed with a saturated aqueous solution of $\mathrm{NH}_{4} \mathrm{Cl}(1 \mathrm{~h})$ and extracted with EtOAc. In all examples, the crude products were estimated to be greater than 95\% pure by ${ }^{1} \mathrm{H}-\mathrm{NMRs}$.

1-Benzyl-4-phenyl-1H-1,2,3-triazole (10a). Using the general procedure $223 \mathrm{mg}(95 \%)$ of the title compound were prepared from benzyl azide $(9 \mathbf{a}, 125 \mu \mathrm{L})$ and phenylacetylene $(112 \mu \mathrm{L})$. Spectroscopic data for 10a were consistent with previously reported data for this compound [19]. ${ }^{1} \mathrm{H}-\mathrm{NMR}$ $\left(\mathrm{CDCl}_{3}\right): \delta 7.80\left(\mathrm{~d}, J=7.0 \mathrm{~Hz}, 2 \mathrm{H}, \mathrm{H}_{\mathrm{Ar}}\right), 7.66(\mathrm{~s}, 1 \mathrm{H}, \mathrm{NCH}=\mathrm{C}), 7.42-7.37\left(\mathrm{~m}, 5 \mathrm{H}, \mathrm{H}_{\mathrm{Ar}}\right), 7.34-7.31$ $\left.\left(\mathrm{m}, 3 \mathrm{H}, \mathrm{H}_{\mathrm{Ar}}\right), 5.59(\mathrm{~s}, 2 \mathrm{H}, \mathrm{PhCH})_{2}\right){ }^{13} \mathrm{C}-\mathrm{NMR}\left(\mathrm{CDCl}_{3}\right): \delta 148.2(\mathrm{C},=\mathrm{C}-\mathrm{Ph}), 134.7\left(\mathrm{C}, \mathrm{C}_{\mathrm{Ar}}\right), 130.5(\mathrm{C}$, $\left.\mathrm{C}_{\mathrm{Ar}}\right), 129.1\left(\mathrm{CH}, \mathrm{CH}_{\mathrm{Ar}}\right), 128.8\left(\mathrm{CH}, \mathrm{CH}_{\mathrm{Ar}}\right), 128.8\left(\mathrm{CH}, \mathrm{CH}_{\mathrm{Ar}}\right), 128.1\left(\mathrm{CH}, \mathrm{CH}_{\mathrm{Ar}}\right), 128.0\left(\mathrm{CH}, \mathrm{CH}_{\mathrm{Ar}}\right)$, $125.7\left(\mathrm{CH}, \mathrm{CH}_{\mathrm{Ar}}\right), 119.5(\mathrm{CH}, \mathrm{NCH}=), 54.2\left(\mathrm{CH}_{2}\right)$.

1-Benzyl-4-butyl-1H-1,2,3-triazole (10b). Using the general procedure $199 \mathrm{mg}(92 \%)$ of the title compound were prepared from benzyl azide (9a, $125 \mu \mathrm{L}), 1$-hexyne $(115 \mu \mathrm{L})$, and $\mathbf{8}(12 \mathrm{mg}, 2 \mathrm{~mol} \%)$ at $40{ }^{\circ} \mathrm{C}$, Spectroscopic data for $\mathbf{1 0 b}$ were consistent with previously reported data for this compound [10]. ${ }^{1} \mathrm{H}-\mathrm{NMR}\left(\mathrm{CDCl}_{3}\right): \delta 7.40-7.34\left(\mathrm{~m}, 3 \mathrm{H}, \mathrm{H}_{\mathrm{Ar}}\right), 7.26-7.24\left(\mathrm{~m}, 2 \mathrm{H}, \mathrm{H}_{\mathrm{Ar}}\right), 7.18(\mathrm{~s}, 1 \mathrm{H}, \mathrm{NCH}=\mathrm{C}), 5.50(\mathrm{~s}$, $2 \mathrm{H}, \mathrm{PhCH} \mathrm{H}_{2} \mathrm{~N}$ ), 2.69 (t, $J=7.5 \mathrm{~Hz}, 2 \mathrm{H}, \mathrm{C}=\mathrm{CNCH}_{2}$ ), 1.62 (quintet, $J=7.5 \mathrm{~Hz}, 2 \mathrm{H}, \mathrm{CH}_{2} \mathrm{CH}_{2} \mathrm{CH}_{2}$ ), 1.37 (sextet, $\left.J=7.3 \mathrm{~Hz}, 2 \mathrm{H}, \mathrm{CH}_{2} \mathrm{CH}_{3}\right), 0.91\left(\mathrm{t}, J=7.3 \mathrm{~Hz}, 3 \mathrm{H}, \mathrm{CH}_{3}\right) .{ }^{13} \mathrm{C}-\mathrm{NMR}\left(\mathrm{CDCl}_{3}\right): \delta 149.8(\mathrm{C}$, $\mathrm{NC}=C$-butyl $), 135.0\left(\mathrm{C}, \mathrm{C}_{\mathrm{Ar}}\right), 129.0\left(\mathrm{CH}, \mathrm{CH}_{\mathrm{Ar}}\right), 128.6\left(\mathrm{CH}, \mathrm{CH}_{\mathrm{Ar}}\right), 127.9\left(\mathrm{CH}, \mathrm{CH}_{\mathrm{Ar}}\right), 120.9(\mathrm{CH}$, $\mathrm{NCH}=)$, $54.0\left(\mathrm{CH}_{2}, \mathrm{PhCH}_{2}\right), 31.4\left(\mathrm{CH}_{2}\right), 25.4\left(\mathrm{CH}_{2}\right), 22.3\left(\mathrm{CH}_{2}\right), 13.8\left(\mathrm{CH}_{3}\right)$.

1-Benzyl-4-(2-hydroxypropan-2-yl)-1H-1,2,3-triazole (10c). Using the general procedure $183 \mathrm{mg}$ $(84 \%)$ of the title compound were prepared from benzyl azide $(9 \mathbf{a}, 125 \mu \mathrm{L})$, 2-methylbut-3-yn-2-ol $(100 \mu \mathrm{L})$, and $\mathbf{8}(6 \mathrm{mg}, 1 \mathrm{~mol} \%)$ at $40{ }^{\circ} \mathrm{C}$. Spectroscopic data for $\mathbf{1 0 c}$ were consistent with previously reported data for this compound [19]. ${ }^{1} \mathrm{H}-\mathrm{NMR}\left(\mathrm{CDCl}_{3}\right): 7.39-7.35\left(\mathrm{~m}, 3 \mathrm{H}, \mathrm{H}_{\mathrm{Ar}}\right), 7.34(\mathrm{~s}, 1 \mathrm{H}$, $\mathrm{NCH}=\mathrm{C}), 7.31-7.27\left(\mathrm{~m}, 2 \mathrm{H}, \mathrm{H}_{\mathrm{Ar}}\right), 5.51\left(\mathrm{~s}, 2 \mathrm{H}, \mathrm{CH}_{2}\right), 2.34($ br s, $1 \mathrm{H}, \mathrm{OH}), 1.61\left(\mathrm{~s}, 6 \mathrm{H}, \mathrm{CH}_{3}\right) .{ }^{13} \mathrm{C}-\mathrm{NMR}$ $\left(\mathrm{CDCl}_{3}\right): \delta 156.0(\mathrm{C}, \mathrm{NC}=\mathrm{C}-), 134.6\left(\mathrm{C}, \mathrm{C}_{\mathrm{Ar}}\right), 129.2\left(\mathrm{CH}, \mathrm{CH}_{\mathrm{Ar}}\right), 128.6,\left(\mathrm{CH}, \mathrm{CH}_{\mathrm{Ar}}\right), 128.2(\mathrm{CH}$, $\left.\mathrm{CH}_{\mathrm{Ar}}\right), 119.1(\mathrm{CH}, \mathrm{NCH}=), 68.3\left(\mathrm{C}, \mathrm{C}(\mathrm{OH}) \mathrm{Me}_{2}\right), 54.2\left(\mathrm{CH}_{2}, \mathrm{PhCH}_{2}\right), 30.5\left(\mathrm{CH}_{3}\right)$. 
1-(2-Diisopropylaminoethyl)-4-phenyl-1H-1,2,3-triazole (10d). Using the general procedure $232 \mathrm{mg}$ $(85 \%)$ of the title compound were prepared from azide $9 \mathrm{~b}(170 \mathrm{mg})$ and phenylacetylene $(112 \mu \mathrm{L})$. ${ }^{1} \mathrm{H}-\mathrm{NMR}\left(\mathrm{CDCl}_{3}\right): \delta 7.84-7.81\left(\mathrm{~m}, 3 \mathrm{H}, \mathrm{H}_{\mathrm{Ar}}\right), 7.45\left(\mathrm{t}, J=6.4 \mathrm{~Hz}, 2 \mathrm{H}, \mathrm{H}_{\mathrm{Ar}}\right), 7.35\left(\mathrm{t}, J=6.4 \mathrm{~Hz}, 1 \mathrm{H}, \mathrm{H}_{\mathrm{Ar}}\right)$, $4.38\left(\mathrm{t}, J=6.5 \mathrm{~Hz}, 2 \mathrm{H}, \mathrm{CH}_{2}\right), 3.04$ (septet, $\left.J=6.5 \mathrm{~Hz}, 2 \mathrm{H}, \mathrm{CH}\left(\mathrm{CH}_{3}\right)_{2}\right), 2.95\left(\mathrm{t}, J=6.5 \mathrm{~Hz}, 2 \mathrm{H}, \mathrm{CH}_{2}\right.$ ), $0.99\left(\mathrm{~d}, J=6.5 \mathrm{~Hz}, 12 \mathrm{H}, \mathrm{CH}_{3}\right) ;{ }^{13} \mathrm{C}-\mathrm{NMR}\left(\mathrm{CDCl}_{3}\right): \delta 147.1(\mathrm{NCH}=\mathrm{C}), 130.9\left(\mathrm{C}, \mathrm{C}_{\mathrm{Ar}}\right), 128.8(\mathrm{CH}$, $\left.\mathrm{CH}_{\mathrm{Ar}}\right), 127.9\left(\mathrm{CH}, \mathrm{CH}_{\mathrm{Ar}}\right), 125.7\left(\mathrm{CH}, \mathrm{CH}_{\mathrm{Ar}}\right), 120.8(\mathrm{CH}, \mathrm{NCH}=), 51.2\left(\mathrm{CH}_{2}, \mathrm{CH}_{2} \mathrm{~N}\right), 48.7(\mathrm{CH}, \mathrm{CHN})$, $45.7\left(\mathrm{CH}_{2}, \mathrm{CH}_{2} \mathrm{~N}\right), 20.8\left(\mathrm{CH}_{3}\right)$. HRMS calculated for $\mathrm{C}_{16} \mathrm{H}_{25} \mathrm{~N}_{4}$ : 273.2079 ; found $273.2086\left[(\mathrm{M}+\mathrm{H})^{+}\right]$.

Ethyl 1-(2-(1,3-dioxolan-2-yl)ethyl)-1H-1,2,3-triazole-4-carboxylate (10e). Using the general procedure $203 \mathrm{mg}$ (84\%) of the title compound were prepared from azide 9c (143 mg), ethyl propiolate $(102 \mu \mathrm{L})$, and $\mathbf{8}(6 \mathrm{mg}, 1 \mathrm{~mol} \%)$. Spectroscopic data for 10e were consistent with previously reported data for this compound [20]. ${ }^{1} \mathrm{H}-\mathrm{NMR}\left(\mathrm{CDCl}_{3}\right): \delta 8.12(\mathrm{~s}, 1 \mathrm{H}, \mathrm{NCH}=\mathrm{C}), 4.93(\mathrm{t}, J=4.0 \mathrm{~Hz}, 1 \mathrm{H}$, OCHO), $4.58\left(\mathrm{t}, J=7.0 \mathrm{~Hz}, 2 \mathrm{H}, \mathrm{CH}_{2} \mathrm{CH}_{2} \mathrm{~N}\right), 4.43\left(\mathrm{q}, J=7.0 \mathrm{~Hz}, \mathrm{OCH}_{2} \mathrm{CH}_{3}\right), 4.03-3.93(\mathrm{~m}, 2 \mathrm{H}$, $\left.\mathrm{OCH}_{2} \mathrm{CH}_{2} \mathrm{O}\right), 3.93-3.83\left(\mathrm{~m}, 2 \mathrm{H}, \mathrm{OCH}_{2} \mathrm{CH}_{2} \mathrm{O}\right), 2.33\left(\mathrm{dt}, J=4.0 ; 7.0 \mathrm{~Hz}, \mathrm{CHCH}_{2} \mathrm{CH}_{2}\right), 1.41$ (t, $\left.J=7.0 \mathrm{~Hz}, 3 \mathrm{H}, \mathrm{OCH}_{2} \mathrm{CH}_{3}\right) .{ }^{13} \mathrm{C}-\mathrm{NMR}\left(\mathrm{CDCl}_{3}\right): \delta 160.2(\mathrm{C}, \mathrm{C}=\mathrm{O}), 139.3\left(\mathrm{C},=\mathrm{C}-\mathrm{CO}_{2} \mathrm{Et}\right), 127.5(\mathrm{CH}$, $\mathrm{CH}=\mathrm{C}), 127.5(\mathrm{CH}, \mathrm{O}-\mathrm{CH}-\mathrm{O}), 64.55\left(\mathrm{CH}_{2}, \mathrm{O}-\mathrm{CH}_{2}-\mathrm{CH}_{2}-\mathrm{O}\right), 64.48\left(\mathrm{CH}_{2}, \mathrm{O}-\mathrm{CH}_{2}-\mathrm{CH}_{2}-\mathrm{O}\right), 60.6$ $\left(\mathrm{CH}_{2}, \mathrm{OCH}_{2}-\mathrm{CH}_{3}\right), 45.0\left(\mathrm{CH}_{2}, \mathrm{CH}_{2}-\mathrm{CH}_{2}-\mathrm{N}\right), 33.2\left(\mathrm{CH}_{2}, \mathrm{CH}_{2}-\mathrm{CH}_{2}-\mathrm{N}\right), 13.7\left(\mathrm{CH}_{3}\right)$.

N,N-Dimethyl-1-(1-phenethyl-1H-1,2,3-triazol-4-yl)methanamine (10f). Using the general procedure $202 \mathrm{mg}(88 \%)$ of the title compound were prepared from (2-azidoethyl)benzene (9d, $147 \mathrm{mg})$, dimethylprop-2-ynylamine $(111 \mu \mathrm{L})$ and $8(6 \mathrm{mg}, 1 \mathrm{~mol} \%)$. Spectroscopic data for $\mathbf{1 0 f}$ were consistent with previously reported data for this compound [20]. ${ }^{1} \mathrm{H}-\mathrm{NMR}\left(\mathrm{CDCl}_{3}\right): \delta 7.31-7.21(\mathrm{~m}, 4 \mathrm{H}$, $\left.\mathrm{H}_{\mathrm{Ar}}+\mathrm{NCH}=\mathrm{C}\right), 7.09\left(\mathrm{~d}, J=7.0 \mathrm{~Hz}, 2 \mathrm{H}, \mathrm{H}_{\mathrm{Ar}}\right), 4.58\left(\mathrm{t}, J=7.0 \mathrm{~Hz}, 2 \mathrm{H}, \mathrm{PhCH}_{2} \mathrm{CH}_{2}\right), 3.57(\mathrm{~s}, 2 \mathrm{H}$, $\left.\mathrm{CH}_{2} \mathrm{NMe}_{2}\right), 3.21\left(\mathrm{t}, J=7.0 \mathrm{~Hz}, 2 \mathrm{H}, \mathrm{PhCH}_{2} \mathrm{CH}_{2}\right), 2.23\left(\mathrm{~s}, 6 \mathrm{H}, \mathrm{NMe}_{2}\right) .{ }^{13} \mathrm{C}-\mathrm{NMR}\left(\mathrm{CDCl}_{3}\right): \delta 144.7(\mathrm{C}$, $\mathrm{NCH}=C), 137.0\left(\mathrm{C}, \mathrm{C}_{\mathrm{Ar}}\right), 128.7\left(\mathrm{CH}, \mathrm{CH}_{\mathrm{Ar}}\right), 128.6\left(\mathrm{CH}, \mathrm{CH}_{\mathrm{Ar}}\right), 126.9\left(\mathrm{CH}, \mathrm{CH}_{\mathrm{Ar}}\right), 122.6(\mathrm{CH}, \mathrm{NCH}=)$, $54.2\left(\mathrm{CH}_{2}, \mathrm{PhCH}_{2} \mathrm{CH}_{2} \mathrm{~N}\right), 51.4\left(\mathrm{CH}_{2}, \mathrm{CH}_{2} \mathrm{NMe}_{2}\right), 44.9\left(\mathrm{CH}_{2}, \mathrm{PhCH}_{2}\right), 36.6\left(\mathrm{CH}_{3}\right)$.

4-Phenyl-1-(3-phenyl-2-propenyl)-1H-1,2,3-triazole (10g). Using the general procedure $250 \mathrm{mg}(96 \%)$ of the title compound were prepared from azide 9e $(159 \mathrm{mg})$ and phenylacetylene $(112 \mu \mathrm{L})$. Spectroscopic data for $\mathbf{1 0 g}$ were consistent with previously reported data for this compound [21]. ${ }^{1} \mathrm{H}-\mathrm{NMR}\left(\mathrm{CDCl}_{3}\right): \delta$ 7.84-7.82 (m, 3H, $\left.\mathrm{H}_{\mathrm{Ar}}+\mathrm{NCH}=\mathrm{C}\right), 7.43-7.39\left(\mathrm{~m}, 4 \mathrm{H}, \mathrm{H}_{\mathrm{Ar}}\right), 7.36-7.32(\mathrm{~m}, 4 \mathrm{H}$, $\left.\mathrm{H}_{\mathrm{Ar}}\right), 6.72(\mathrm{~d}, J=16.0 \mathrm{~Hz}, 1 \mathrm{H}, \mathrm{PhCH}=\mathrm{CH}), 6.40(\mathrm{dt}, J=16.0,6.5 \mathrm{~Hz}, 1 \mathrm{H}, \mathrm{PhCH}=\mathrm{CH}), 5.20(\mathrm{~d}, J=6.5 \mathrm{~Hz}$, $\left.2 \mathrm{H}, \mathrm{CH}_{2} \mathrm{~N}\right) .{ }^{13} \mathrm{C}-\mathrm{NMR}\left(\mathrm{CDCl}_{3}\right): \delta 148.0(\mathrm{C}, \mathrm{NCH}=\mathrm{C}), 135.4\left(\mathrm{C}, \mathrm{C}_{\mathrm{Ar}}\right), 135.2\left(\mathrm{CH}, \mathrm{CH}_{\mathrm{Ar}}\right), 130.5(\mathrm{C}$, $\left.\mathrm{C}_{\mathrm{Ar}}\right), 128.7\left(\mathrm{CH}, \mathrm{CH}_{\mathrm{Ar}}\right), 128.6\left(\mathrm{CH}, \mathrm{CH}_{\mathrm{Ar}}\right), 128.4\left(\mathrm{CH}, \mathrm{CH}_{\mathrm{Ar}}\right), 128.0\left(\mathrm{CH}, \mathrm{CH}_{\mathrm{Ar}}\right), 126.6(\mathrm{CH}, \mathrm{PhCH}=)$, 125.6 $(\mathrm{CH}, \mathrm{PhCH}=\mathrm{CH}), 121.8\left(\mathrm{CH}, \mathrm{CH}_{\mathrm{Ar}}\right), 119.3(\mathrm{CH}, \mathrm{NCH}=), 52.3\left(\mathrm{CH}_{2}, \mathrm{CH}_{2} \mathrm{~N}\right)$.

Dimethyl(1-phenyl-1H-1,2,3-triazol-4-ylmethyl)amine (10h). Using the general procedure $196 \mathrm{mg}$ (97\%) of the title compound were prepared from phenyl azide (9f, $107 \mu \mathrm{L})$ and dimethylprop-2ynylamine $(111 \mu \mathrm{L})$. Spectroscopic data for $\mathbf{1 0 h}$ were consistent with previously reported data for this compound [22]. ${ }^{1} \mathrm{H}-\mathrm{NMR}\left(\mathrm{CDCl}_{3}\right): \delta 7.95(\mathrm{~s}, 1 \mathrm{H}, \mathrm{NCH}=\mathrm{C}), 7.74\left(\mathrm{~d}, J=8.0 \mathrm{~Hz}, 2 \mathrm{H}, \mathrm{H}_{\mathrm{Ar}}\right), 7.53(\mathrm{t}$, $\left.J=8.0,2 \mathrm{H}, \mathrm{H}_{\mathrm{Ar}}\right), 7.43\left(\mathrm{t}, J=8.0 \mathrm{~Hz}, 1 \mathrm{H}, \mathrm{H}_{\mathrm{Ar}}\right), 3.71\left(\mathrm{~s}, 2 \mathrm{H}, \mathrm{CH}_{2} \mathrm{NMe}_{2}\right), 2.34\left(\mathrm{~s}, 6 \mathrm{H}, \mathrm{NMe}_{2}\right) .{ }^{13} \mathrm{C}-\mathrm{NMR}$ $\left(\mathrm{CDCl}_{3}\right): \delta 146.0(\mathrm{C}, \mathrm{NCH}=C), 137.0\left(\mathrm{C}, \mathrm{C}_{\mathrm{Ar}}\right), 129.6\left(\mathrm{CH}, \mathrm{CH}_{\mathrm{Ar}}\right), 128.5\left(\mathrm{CH}, \mathrm{CH}_{\mathrm{Ar}}\right), 120.4(\mathrm{CH}$, $\mathrm{NCH}=), 120.3\left(\mathrm{CH}, \mathrm{CH}_{\mathrm{Ar}}\right), 54.3\left(\mathrm{CH}_{2}, \mathrm{CH}_{2} \mathrm{~N}\right), 45.2\left(\mathrm{CH}_{3}\right)$. 
6-(4-Phenyl-1,2,3-triazol-1-yl)hexanenitrile (10i). Using the general procedure $227 \mathrm{mg}(94 \%)$ of the title compound were prepared from 6-azidohexanenitrile $(9 \mathrm{~g}, 138 \mathrm{mg})$ and phenylacetylene $(112 \mu \mathrm{L})$. Spectroscopic data for 10i were consistent with previously reported data for this compound [23]. ${ }^{1} \mathrm{H}-\mathrm{NMR}\left(\mathrm{CDCl}_{3}\right): \delta$ 7.85-7.82 (m, 2H, $\left.\mathrm{H}_{\mathrm{Ar}}\right), 7.76(\mathrm{~s}, 1 \mathrm{H}, \mathrm{NCH}=), 7.45-7.42\left(\mathrm{~m}, 2 \mathrm{H}, \mathrm{H}_{\mathrm{Ar}}\right), 7.36-7.31$ $\left(\mathrm{m}, 1 \mathrm{H}, \mathrm{H}_{\mathrm{Ar}}\right), 4.44\left(\mathrm{t}, J=7.0 \mathrm{~Hz}, 2 \mathrm{H}, \mathrm{CH}_{2} \mathrm{~N}\right), 2.36\left(\mathrm{t}, J=7.0 \mathrm{~Hz}, 2 \mathrm{H}, \mathrm{CH}_{2} \mathrm{CN}\right), 2.08-1.97$ (m, $\left.2 \mathrm{H}, \mathrm{CH}_{2}\right), 1.77-1.67\left(\mathrm{~m}, 2 \mathrm{H}, \mathrm{CH}_{2}\right), 1.59-1.48\left(\mathrm{~m}, 2 \mathrm{H}, \mathrm{CH}_{2}\right) .{ }^{13} \mathrm{C}-\mathrm{NMR}\left(\mathrm{CDCl}_{3}\right): \delta 147.8(\mathrm{C}, \mathrm{C}=\mathrm{C}-\mathrm{Ph})$, $130.5\left(\mathrm{C}, \mathrm{C}_{\mathrm{Ar}}\right), 128.8\left(\mathrm{CH}, \mathrm{C}_{\mathrm{Ar}}\right), 128.1\left(\mathrm{CH}, \mathrm{C}_{\mathrm{Ar}}\right), 125.6\left(\mathrm{CH}, \mathrm{C}_{\mathrm{Ar}}\right), 119.5(\mathrm{CH}, \mathrm{CH}=\mathrm{C}-\mathrm{Ph}), 119.3(\mathrm{C}$, $\mathrm{CN}), 49.8\left(\mathrm{CH}_{2}, \mathrm{CH}_{2}-\mathrm{N}\right), 29.5\left(\mathrm{CH}_{2}\right), 25.5\left(\mathrm{CH}_{2}\right), 24.7\left(\mathrm{CH}_{2}\right), 17.0\left(\mathrm{CH}_{2}\right)$.

4-Cyclopropyl-1-(1-phenylethyl)-1H-1,2,3-triazole (10j). Using the general procedure $183 \mathrm{mg}(85 \%)$ of the title compound were prepared from azide $\mathbf{9 h}(147 \mathrm{mg})$, ethynylcyclopropane $(87 \mu \mathrm{L})$ and $\mathbf{8}$ $(6 \mathrm{mg}, 1 \mathrm{~mol} \%)$ at $40{ }^{\circ} \mathrm{C}$. Spectroscopic data for $\mathbf{1 0 j}$ were consistent with previously reported data for this compound [23]. ${ }^{1} \mathrm{H}-\mathrm{NMR}\left(\mathrm{CDCl}_{3}\right): \delta 7.40-7.28\left(\mathrm{~m}, 3 \mathrm{H}, \mathrm{H}_{\mathrm{Ar}}\right), 7.27-7.24\left(\mathrm{~m}, 2 \mathrm{H}, \mathrm{H}_{\mathrm{Ar}}\right), 7.11(\mathrm{~s}, 1 \mathrm{H}$, $\mathrm{NCH}=\mathrm{C}), 5.76(\mathrm{q}, 1 \mathrm{H}, J=7.0 \mathrm{~Hz}, \mathrm{PhCH}), 1.95\left(\mathrm{~d}, 3 \mathrm{H}, J=7.0 \mathrm{~Hz}, \mathrm{CH}_{3}\right), 1.93-1.87(\mathrm{~m}, 1 \mathrm{H}$, $\left.\mathrm{CH}_{\text {cyclopropyl }}\right), \quad 0.94-0.87\left(\mathrm{~m}, 2 \mathrm{H}, \mathrm{CH}_{2}\right), 0.84-0.79\left(\mathrm{~m}, 2 \mathrm{H}, \mathrm{CH}_{2}\right) .{ }^{13} \mathrm{C}-\mathrm{NMR}\left(\mathrm{CDCl}_{3}\right): \delta 150.2$ $\left(\mathrm{C}=\mathrm{C}_{\text {cyclopropyl }}\right), 140.1\left(\mathrm{C}, \mathrm{C}_{\mathrm{Ar}}\right), 128.9\left(\mathrm{CH}, \mathrm{CH}_{\mathrm{Ar}}\right), 128.3\left(\mathrm{CH}, \mathrm{CH}_{\mathrm{Ar}}\right), 126.4\left(\mathrm{CH}, \mathrm{CH}^{\mathrm{Ar}}\right), 118.3(\mathrm{CH}$, $\mathrm{N}-\mathrm{CH}=\mathrm{C}), 58.9(\mathrm{CH}, \mathrm{PhCH}), 21.2\left(\mathrm{CH}_{3}\right), 7.6\left(\mathrm{CH}, \mathrm{CH}_{\text {cyclopropyl }}\right), 6.7\left(\mathrm{CH}_{2}, \mathrm{CH}_{2}\right)$.

\section{Conclusions}

Diimine copper(I) complexes are highly efficient catalysts for the [3+2] cycloaddition of azides and terminal alkynes. Low copper loadings were enough to ensure high isolated yields of 1,2,3-triazoles at room temperature, in air and in technical grade acetone. Furthermore, purification by column chromatography was not necessary, and a simple extraction was enough to isolate pure triazoles. The reported catalysts are noticeably more active than related Ar-BIAN complexes. This might be attributed to two factors: (1) the presence of alkyl groups on the diimine ligands; which in these series systematically outperformed aryl diimines; or (2) the increased chemical stability of simple diimine scaffolds, in particular towards oxidation and reduction reactions.

\section{Acknowledgments}

Imperial College London is acknowledged for financial support. J.M.B. thanks the Universidad de Barcelona for an Erasmus Scholarship.

\section{Conflict of Interest}

The authors declare no conflict of interest.

\section{References}

1. Tornøe, C.W.; Christensen, C.; Meldal, M. Peptidotriazoles on solid phase: [1,2,3]-Triazoles by regiospecific copper(I)-catalyzed 1,3-dipolar cycloaddition of terminal alkynes to azides. J. Org. Chem. 2002, 67, 3057-3064. 
2. Rostovtsev, V.V.; Green, L.G.; Fokin, V.V.; Sharpless, K.B. A stepwise huisgen cycloaddition process: Copper(I)-catalyzed regioselective "ligation" of azides and terminal alkynes. Angew. Chem. Int. Ed. 2002, 41, 2596-2599.

3. L'abbe, G. Are azidocumulenes accessible? Bull. Soc. Chem. Belg. 1984, 93, 579-592.

4. Special Issue on Click Chemistry. Chem. Soc. Rev. 2010, 39, 1221-1408.

5. Kolb, H.C.; Finn, M.G.; Sharpless, K.B. Click chemistry: Diverse chemical function from a few good reactions. Angew. Chem. Int. Ed. 2001, 40, 2004-2021.

6. Díez-González, S. Well-defined copper(I) complexes for Click azide-alkyne cycloaddition reactions: One Click beyond. Catal. Sci. Tech. 2011, 1, 166-178.

7. Donnelly, P.S.; Zanatta, S.D.; Zammit, S.C.; White, J.M.; Williams, S.J. "Click” cycloaddition catalysts: Copper(I) and copper(II) tris(triazolylmethyl)amine complexes. Chem. Commun. 2008, 2008, 2459-2461.

8. Chan, T.R.; Fokin, V.V. Polymer-supported copper(i) catalysts for the experimentally simplified azide-alkyne cycloaddition. QSAR Comb. Sci. 2007, 26, 1274-1279.

9. Özçubukçu, S.; Ozkal, E.; Jimeno, C.; Pericàs, M.A. A highly active catalyst for Huisgen 1,3-dipolar cycloadditions based on the tris(triazolyl)methanol-Cu(I) structure. Org. Lett. 2009, 11, 4680-4683.

10. Candelon, N.; Lastécouères, D.; Diallo, A.K.; Aranzaes, J.R.; Astruc, D.; Vincent, J.M. A highly active and reusable copper(I)-tran catalyst for the "click" 1,3-dipolar cycloaddition of azides and alkynes. Chem. Commun. 2008, 2008, 741-743.

11. Li, L.; Lopez, P.S.; Rosa, V.; Figueira, C.A.; Lemos, M.A.N.D.A.; Duarte, M.T.; Avilés, T.; Gomes, P.T. Synthesis and structural characterisation of (aryl-BIAN)copper(I) complexes and their application as catalysts for the cycloaddition of azides and alkynes. Dalton Trans. 2012, 41, 5144-5154.

12. Frutos Pedreño, R.; Markalain-Barta, J.; Vega Isa, E.; Díez-González, S. Manuscript in preparation, 2013.

13. Alvarez, S.G.; Alvarez, M.T. A practical procedure for the synthesis of alkyl azides at ambient temperature in demethylsulfoxide in high purity and yield. Synthesis 1997, 1997, 413-414.

14. Engler, A.C.; Bonner, D.K.; Buss, H.G.; Cheung, E.Y.; Hammond, P.T. The synthetic tuning of clickable $\mathrm{pH}$ responsive cationic polypeptides and block copolypeptides. Soft Matter 2011, 7, 5627-5637.

15. Carboni, B.; Vaultier M.; Carrié, R. Etude de la chimioselectivite de la reaction des dichloroboranes avec les azides fonctionnels: une synthese efficace d'amines secondaires fonctionnalisees. Tetrahedron 1987, 43, 1799-1810.

16. Van Kalkeren, H.A.; Bruins, J.J.; Rutjes, F.P.J.T.; van Delft, F.L. Organophosphorus-catalysed staudinger reduction. Adv. Synth. Catal. 2012, 354, 1417-1421.

17. Rehse, K.; Cwiklicki, A. Antiaggregating and antithrombotic activities of new 1,2,3triazolecarboxamides. Arch. Pharm. Pharm. Med. Chem. 2004, 337, 156-163.

18. Luo, L.; Wilhelm, C.; Sun, A.; Grey, C.P.; Lauher, J.W.; Goroff, N.S. Poly(diiododiacetylene): Preparation, isolation, and full characterization of a very simple poly(diacetylene). J. Am. Chem.

Soc. 2008, 130, 7702-7709. 
19. Appukkuttan, P.; Dehaen, W.; Fokin, V.V.; van der Eycken, E. A microwave-assisted Click chemistry synthesis of 1,4-disubstituted 1,2,3-triazoles via a copper(I)-catalyzed three-component reaction. Org. Lett. 2004, 6, 4223-4225.

20. Díez-González, S.; Nolan, S.P. $\left[(\mathrm{NHC})_{2} \mathrm{Cu}\right] \mathrm{X}$ complexes as efficient catalysts for azide-alkyne Click chemistry at low catalyst loadings. Angew. Chem. Int. Ed. 2008, 47, 8881-8884.

21. Kamijo, S.; Jin, T.; Huo, Z.; Yamamoto, Y. A one-pot procedure for the regioconrolled synthesis of allyltriazoles via the $\mathrm{Pd}-\mathrm{Cu}$ bimetallic catalysed three-component coupling reaction of nonactivated terminal alkynes, allyl carbonate, and trimethylsilyl azide. J. Org. Chem. 2004, 69, 2386-2393.

22. Yan, Z.Y.; Zhao, Y.B.; Fan, M.J.; Liu, W.M.; Liang, Y.M. General synthesis of (1-substituted$1 H$-1,2,3-triazol-4-ylmethyl)-dialkylamines via a copper(I)-catalyzed three-component reaction in water. Tetrahedron 2005, 61, 9331-9337.

23. Lal, S.; Díez-González, S. [CuBr$\left.\left(\mathrm{PPh}_{3}\right)_{3}\right]$ for azide-alkyne cycloaddition reactions under strict Click conditions J. Org. Chem. 2011, 76, 2367-2373.

Sample Availability: Not available.

(C) 2013 by the authors; licensee MDPI, Basel, Switzerland. This article is an open access article distributed under the terms and conditions of the Creative Commons Attribution license (http://creativecommons.org/licenses/by/3.0/). 